\title{
ATAD2 Overexpression Identifies Colorectal Cancer Patients with Poor Prognosis and Drives Proliferation of Cancer Cells
}

\author{
Yang Luo, Guang-Yao Ye, Shao-Lan Qin, Min-Hao Yu, Yi-Fei Mu, and Ming Zhong \\ Department of Gastrointestinal Surgery, Renji Hospital, School of Medicine, Shanghai Jiao Tong University, Shanghai 200127, China \\ Correspondence should be addressed to Ming Zhong; drzhongming@hotmail.com
}

Received 10 May 2015; Accepted 20 September 2015

Academic Editor: Miroslav Zavoral

Copyright ( 2015 Yang Luo et al. This is an open access article distributed under the Creative Commons Attribution License, which permits unrestricted use, distribution, and reproduction in any medium, provided the original work is properly cited.

\begin{abstract}
ATPase family AAA domain-containing 2 (ATAD2) has been identified as a critical modulator involved in cell proliferation and invasion. The purpose of this study was to explore the expression of ATAD2 in CRC tissues as well as its relationship with degree of malignancy. Data containing three independent investigations from Oncomine database demonstrated that ATAD2 is overexpressed in CRC compared with normal tissue, and similar result was also found in 32 pairs of CRC tissues by qPCR. The protein expression of ATAD2 was examined in six CRC cell lines and 300 CRC specimens. The results showed that high expression of ATAD2 was significantly correlated with tumor size $(P<0.001)$, serum CEA $(P=0.012)$, lymph node metastasis $(P=0.018)$, liver metastasis $(P=0.025)$, and clinical stage $(P=0.004)$. Kaplan-Meier method suggested that higher ATAD2 protein expression significantly associated with the overall survival (OS) of CRC patients $(P<0.001)$ and was an independent predictor of poor OS. Functional studies showed that suppression of ATAD2 expression with siRNA could significantly inhibit the growth in SW480 and HCT116 cells. These results indicated that ATAD2 could serve as a prognostic marker and a therapeutic target for CRC.
\end{abstract}

\section{Introduction}

Colorectal cancer (CRC) is one of the most common lethal malignancies in terms of both incidence and mortality [1]. Although the diagnosis and treatment of CRC have been improved, the efficacy of surgery and chemotherapy remains unsatisfactory due to late diagnosis [2]. Therefore, new diagnostic and treatment strategies are urgently needed for this malignancy.

ATPase family AAA domain-containing 2 (ATAD2), also known as ANCCA (AAA+ nuclear coregulator cancer associated), is a novel member of the AAA+ ATPase family $[3,4]$. ATAD2 contains both a bromodomain and an ATPase domain and also maps to chromosome $8 \mathrm{q} 24$ that is the most commonly amplified region in many types of cancer [5]. The especial structure of ATAD2 indicates that it is associated with genome regulation, including cell proliferation, division, apoptosis, and differentiation [6-10]. Recently, it is reported that aberrant expression of ATAD2 contributes to hepatocellular carcinoma proliferation and metastasis $[11,12]$. Studies have revealed that ATAD2 is highly expressed in several types of tumors such as breast cancer, lung cancer, and gastric cancer [13-15]. Thus, ATAD2 manifests oncogenic function and plays a significant role in cancer development. However, the expression of the ATAD2 protein in CRC and its significance remain uncertain.

\section{Methods}

2.1. In Silico Analysis Using the Oncomine Database. To determine the expression pattern of ATAD2 in CRC, three datasets (Kaiser Colon, Hong Colorectal, and Hong Colorectal) in Oncomine database (https://www.oncomine.org) were used. We compared ATAD2 gene expression in CRC tissues with normal colorectal tissues according to the standard procedures as previously described [16].

2.2. Cell Culture and Transfection. Five human CRC cell lines (HCT116, SW480, LoVo, T84, and HT29) and a normal control colon cell line (HCoEpiC) were all preserved in Shanghai Cancer Institute. All of these cells lines were cultured in DMEM medium (Invitrogen) supplemented with 
$10 \%(\mathrm{v} / \mathrm{v})$ fetal bovine serum (FBS) and $1 \%$ antibiotics at $37^{\circ} \mathrm{C}$ in a humidified incubator under $5 \% \mathrm{CO}_{2}$ condition.

The transfections were performed using Lipofectamine 2000 (Invitrogen, USA). Small interfering RNAs (siRNA) targeting ATAD2 and a negative control were obtained from GenePharma Technology (Shanghai, China). The transfection was performed according to the manufacturer's protocol.

2.3. Patients and Tissue Samples. A total of 300 formalinfixed paraffin-embedded CRC tissues were collected to perform immunohistochemical staining from January 2005 to November 2014 at the Renji Hospital, Shanghai Jiao Tong University School of Medicine, China. Moreover, additional 32 snap-frozen CRC tissues and corresponding adjacent noncancerous tissues to isolate RNA, which were also obtained from Renji Hospital, were enrolled in this study simultaneously. Inclusion criteria were histologically confirmed CRC and curative resection of tumor without preoperative or postoperative adjuvant therapy. Important clinical data, such as tumor location, serum CEA level, and clinical stage, were collected from each patient's medical records. The follow-up time was calculated from the date of surgery to the date of death, or the last known follow-up. All CRC tissue samples in this study were obtained with patients' written informed consent and all experiments have been approved by the ethics committee at local hospital.

2.4. Real-Time Quantitative PCR. Total RNA from primary tumor and adjacent noncancerous tissue samples was extracted using Trizol reagent (Takara, Japan), and according to the manufacturer's instructions, reverse transcription was performed by PrimeScript RT-PCR kit (Takara, Japan). Real-time quantitative PCR (qPCR) was performed using a 7500 real-time PCR system (Applied Biosystems, Inc., USA). The primers for ATAD2 were as follows: forward: 5'-GGAATCCCAAACCACTGGACA-3'; reverse: $5^{\prime}$-GGTAGCGTCGTCGTAAAGCACA-3'. GAPDH mRNA was used to standardize the relative expression of ATAD2. The primers for GAPDH were as follows: forward: $5^{\prime}$-GCATTGCCCTCAACGACCAC-3', reverse: $5^{\prime}$-CCACCACCCTGTTGCTGTAG-3'.

2.5. Immunohistochemical Staining. Four-micrometer-thick tissue sections were subjected to immunohistochemical staining with avidin-biotin-peroxidase complex system which was performed as previously described [17]. Tissue sections were incubated by anti-ATAD2 antibody $(1: 400$, Abcam, Cambridge, UK) at $4^{\circ} \mathrm{C}$ overnight. Immunohistochemical staining was scored by two independent pathologists according to intensity and percentage of positive cells simultaneously. Staining intensity was scored as follows: 0 : negative; 1 : weak staining; 2 : moderate staining; 3: strong staining, and the percentage of positive cells was scored on a scale of $0-4(0,<5 \% ; 1,5 \%-30 \% ; 2,30 \%-50 \% ; 3$, $51 \%-75 \% ; 4,>75 \%)$. And the final score was designated as low or high expression group using the percent of positive cell score $\times$ staining intensity score as follows: low expression was defined as a total score $<6$ and high expression with a total score $\geq 6$.
2.6. Western Blot. Western blot was performed as previously described [18]. Cells were harvested after 48-hour transfection, and $40 \mu \mathrm{g}$ of protein samples was resolved by SDS-PAGE and transferred to PVDF membranes. The membranes were probed with the same primary antibody used in immunostaining overnight at $4^{\circ} \mathrm{C}$ and incubated for 1 hour with secondary antibody (Boston, MA, USA). The Western blotting analysis was repeated at least three times.

2.7. Cell Proliferation Assay. Cell viability was assessed by the Cell Counting Kit 8 (CCK-8; Dojindo). Briefly, control and treated SW480 and HCT116 cells were seeded into 96well plates at an initial density of 3000 cells/well. At each time point, $10 \mu \mathrm{L}$ of CCK- 8 solution was added to each well and incubated for 2 hours. The absorbance was measured by scanning with a microplate reader at $450 \mathrm{~nm}$. The experiment was repeated at least three times.

2.8. Statistical Analysis. Statistical analyses were performed by SPSS 19.0 (SPSS Inc; Chicago, USA). The expression of ATAD2 mRNA in CRC tissues and corresponding noncancerous tissues was analyzed with the Student's $t$-test. The differences of the relative absorbance value of CCK8 assays also were determined using Student's $t$-test. The Chi-square test was used to analyze the relationship between ATAD2 expression and clinicopathological features. Survival rate was evaluated by Kaplan-Meier method and differences between survival curves were tested by the log-rank test. $P$ values less than 0.05 were considered to be statistically significant.

\section{Results}

3.1. ATAD2 Is Overexpressed in CRC at mRNA Level. To roundly investigate ATAD2 expression in CRC, we analyzed three independent microarray datasets from Oncomine database [19-21]. The results showed that the mRNA expression levels of ATAD2 were upregulated in most of CRC tissues, compared with normal tissue (Figures 1(a)-1(c)). Then, 32 pairs of CRC and matched normal tissues were subjected to qPCR. Consistent with the data from Oncomine database, ATAD2 was also overexpressed in $68.75 \%(22 / 32)$ of CRC patients at mRNA level (Figure $1(\mathrm{~d})$ ).

3.2. ATAD2 Is Expressed Diversely in CRC at Protein Level. To further investigate the expression of ATAD2 at the protein level, we measured ATAD2 level in CRC cell lines and tissues. The expression of ATAD2 protein was increased in all five CRC cell lines compared with the nonmalignant $\mathrm{HCoEpiC}$ cells. Moreover, we found that the higher expression of ATAD2 protein is in low differentiated CRC cell lines (SW480, HCT116, and LoVo) compared with welldifferentiated cell lines (HT29, T84) (Figures 1(e)-1(f)). Then, we tested $300 \mathrm{CRC}$ tissue samples by using the method of immunohistochemical staining and found that ATAD2 was low expressed in the 124 (41.33\%) of the total 300 CRC samples while the remaining 176 (58.67\%) samples remained at a high expression level (Figures 2(a)-2(c)). 


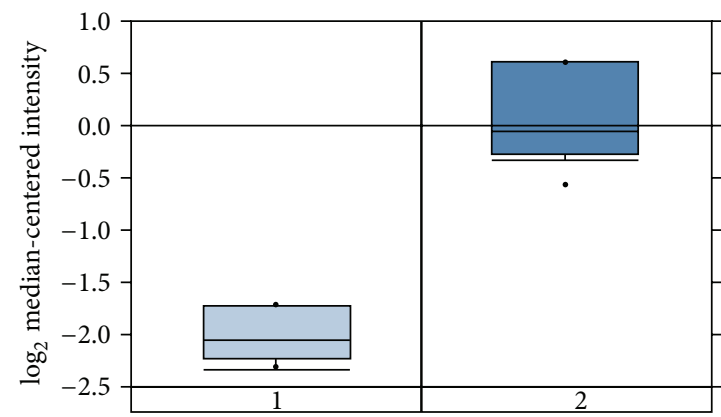

(1) Colon (5)

(2) Rectosigmoid adenocarcinoma (10)

(a)

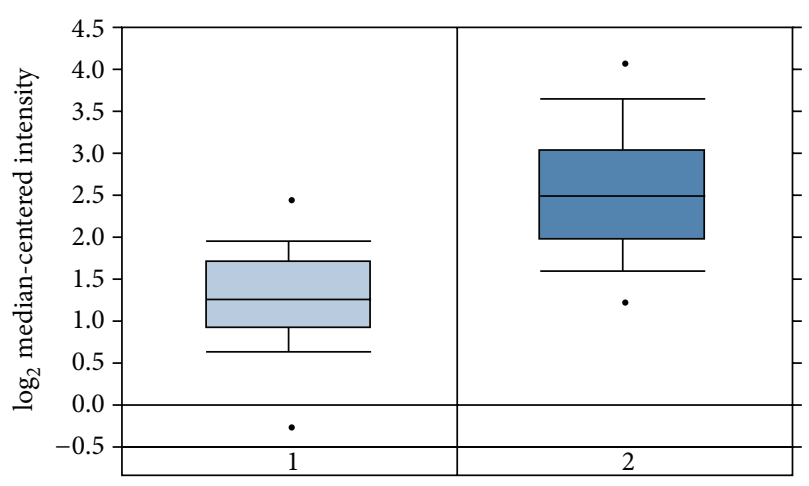

(1) Colorectal tissue (24)

(2) Colorectal carcinoma (36)

(c)

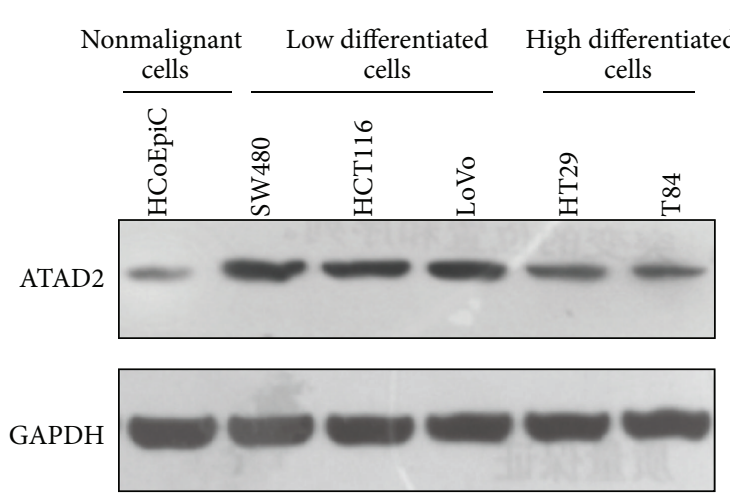

(e)

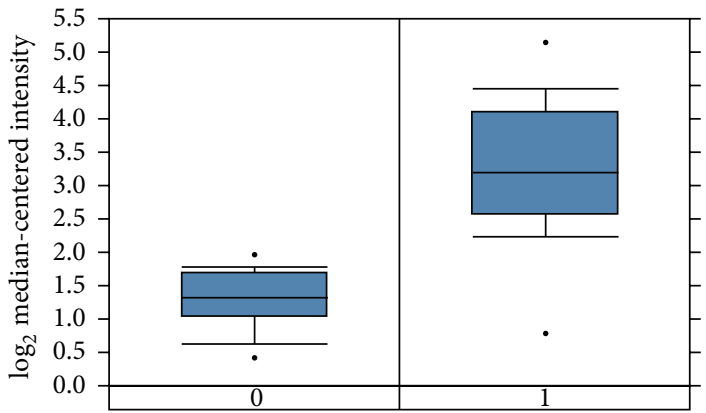

(0) No value (12)

(1) Colorectal carcinoma (70)

(b)

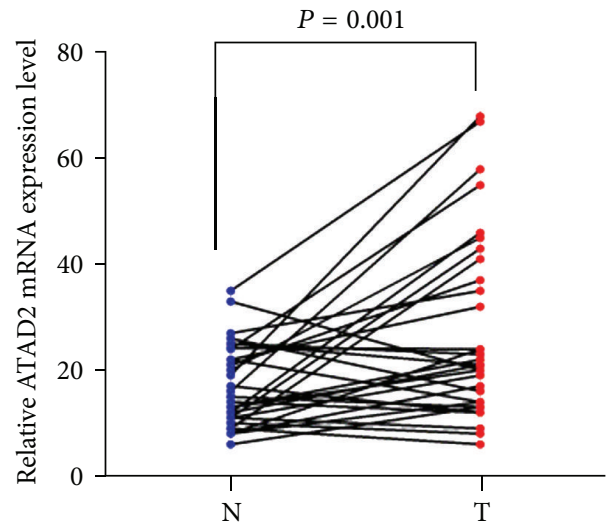

(d)

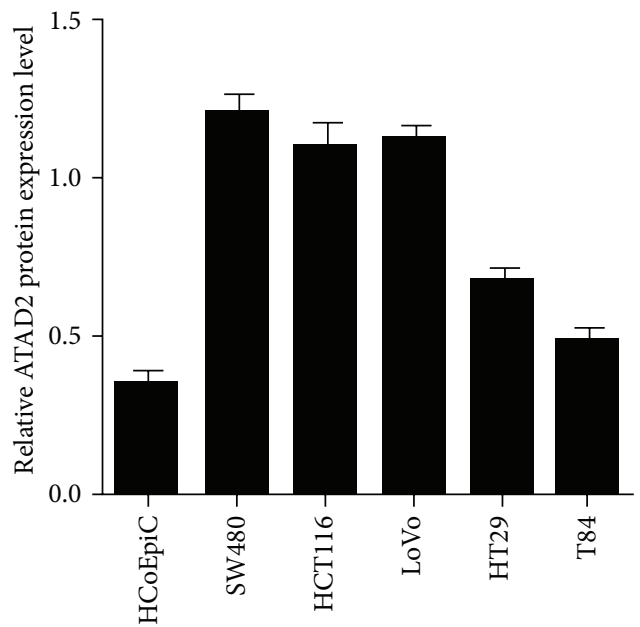

(f)

Figure 1: ATAD2 expression in CRC at mRNA and protein level. (a)-(c) ATAD2 expression in Kaiser Colon (a), Hong Colorectal, (b) and Skrzypczak Colorectal (c). (d) Increased ATAD2 mRNA expression in 32 matched tumor (T) and nontumor tissues (N) was detected by qPCR. (e)-(f) Western blots show the ATAD2 expression in five CRC cell lines and the nonmalignant HCoEpiC cells. $P$ values were calculated by paired $t$-test. 


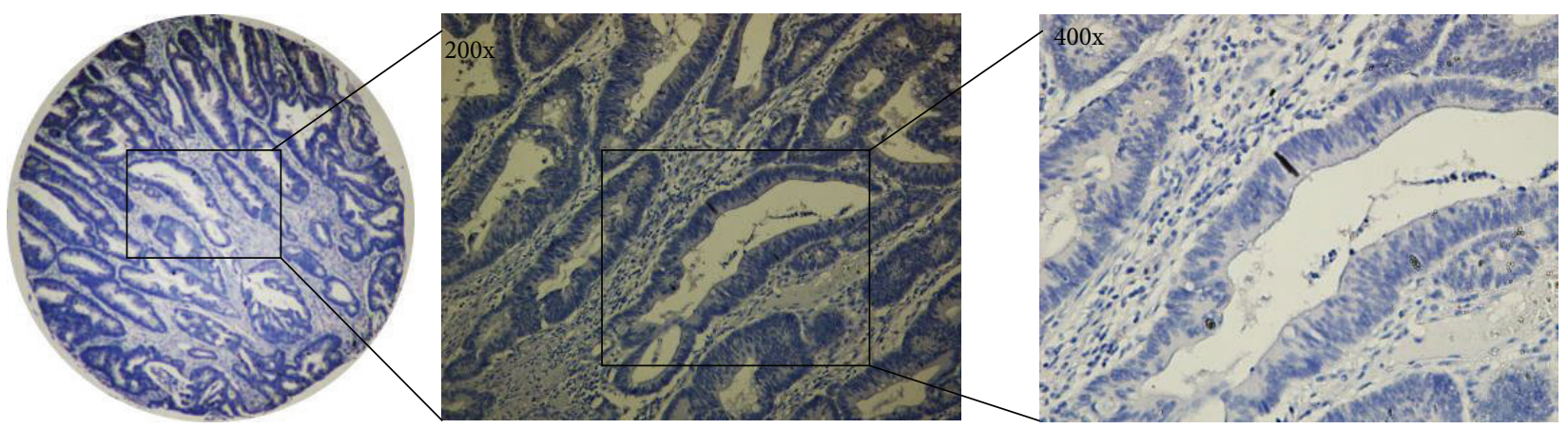

ATAD2 negative expression

(a)

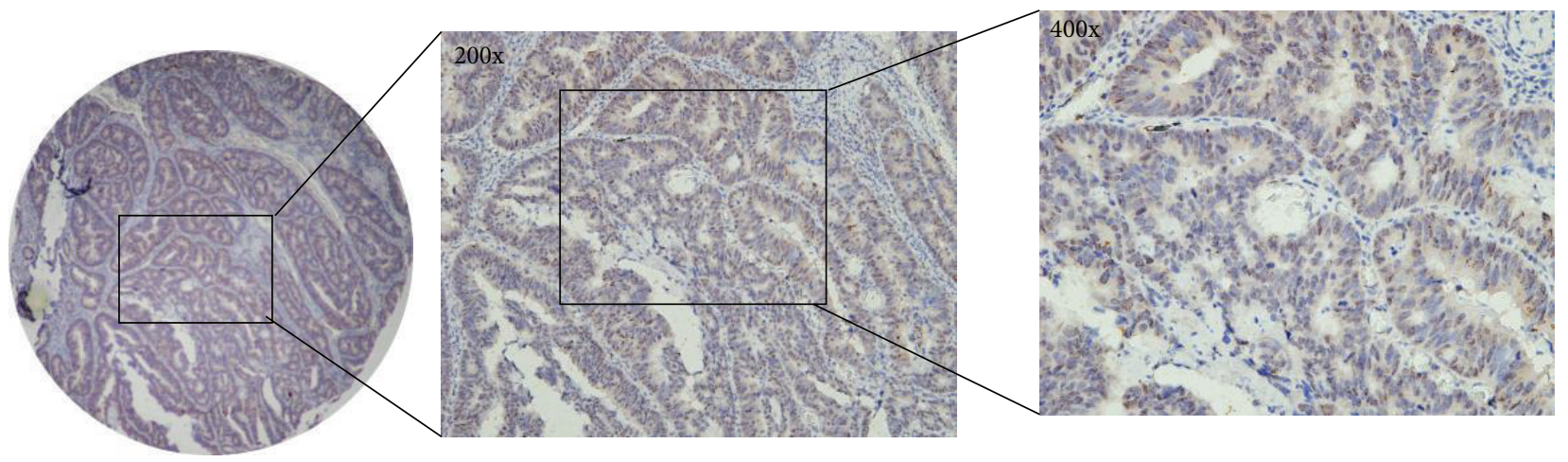

ATAD2 low expression

(b)

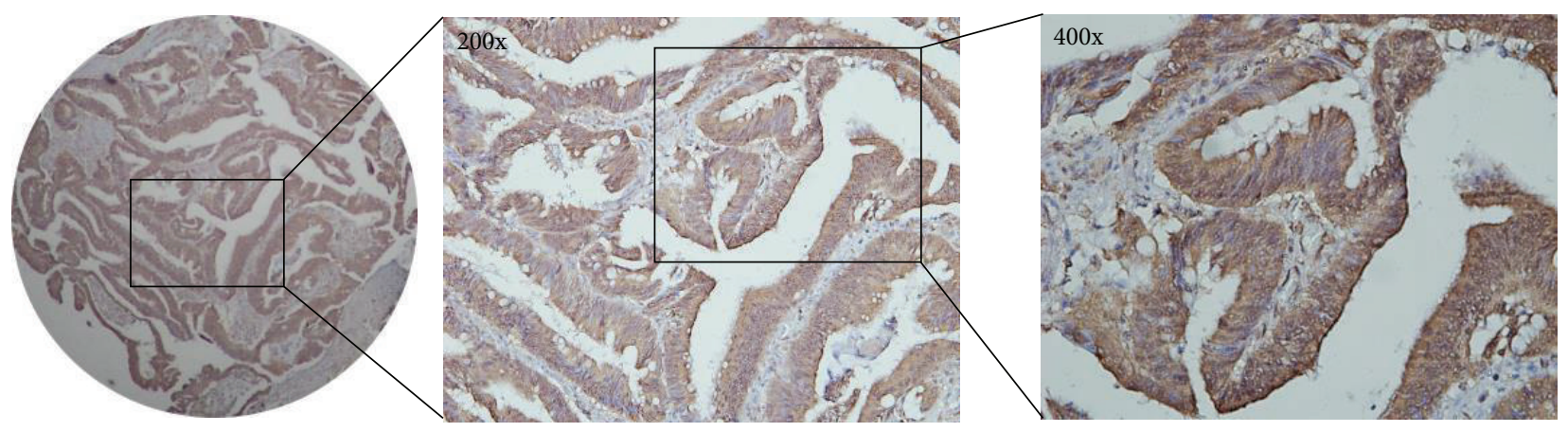

ATAD2 high expression

(c)

FIgURE 2: ATAD2 expression in CRC was determined by immunochemistry. (a) Negative expression of ATAD2, (b) low expression of ATAD2, and (c) high expression of ATAD2. Representative images are shown at 200x and 400x magnification, respectively.

3.3. Relationship between ATAD2 Expression and Corresponding Clinical Parameters. To evaluate the clinical significance of ATAD2, the Chi-square test was used to analyze correlations between ATAD2 protein expression and clinicopathological parameters in CRC. The results indicated that high expression of ATAD2 in CRC tissues is closely correlated with tumor size $(P<0.001)$, serum CEA level $(P=0.012)$, lymph node metastasis $(P=0.018)$, liver metastasis $(P=0.025)$, and clinical stage $(P=0.004)$. However, no statistically significant correlations were identified between ATAD2 expression and other clinicopathologic characteristics, including age, gender, and tumor location (Table 1).

3.4. Correlation between ATAD2 Expression and Prognosis in CRC Patients. To investigate the prognostic influence of ATAD2, the overall survival (OS) rate of CRC patients was analyzed using Kaplan-Meier survival curves and the logrank test. The result revealed that high expression of ATAD2 was inversely associated with OS for all 189 samples $(P<$ 0.001) (Figure 3(a)). In addition, The OS of ATAD2 negative 


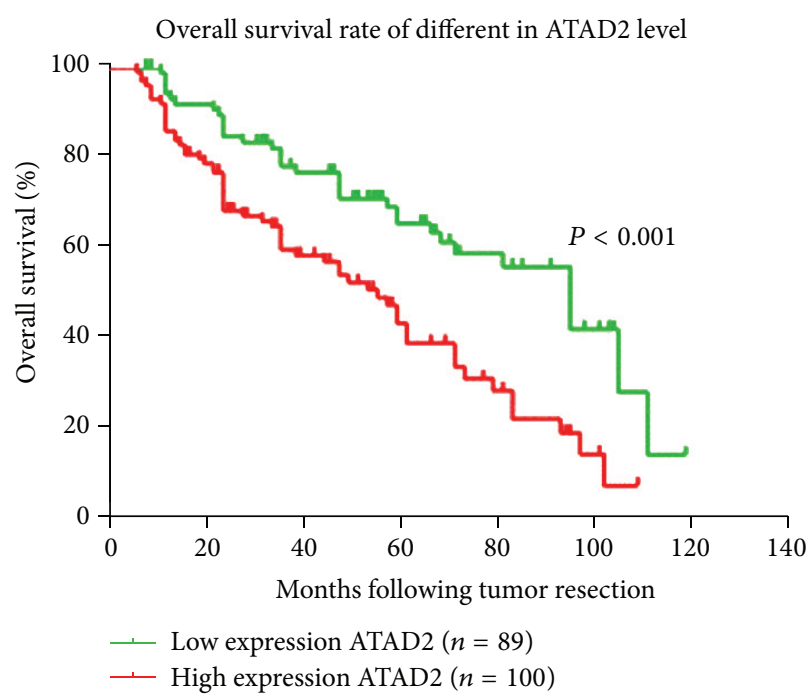

(a)

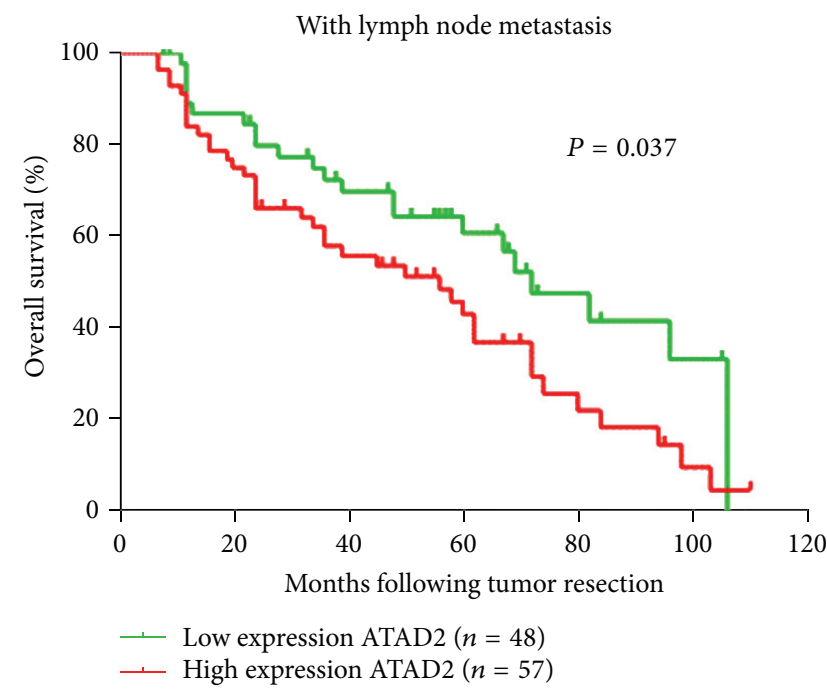

(c)

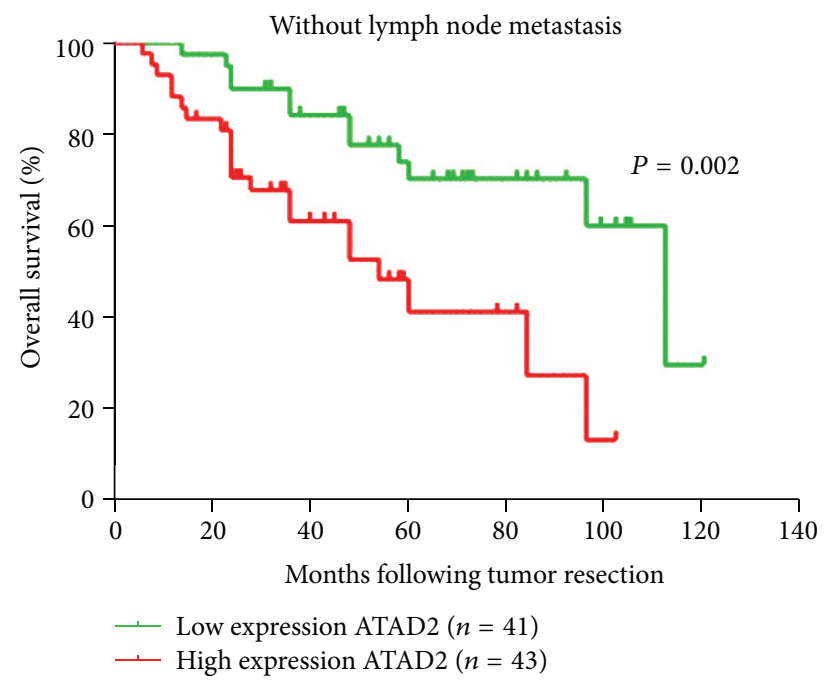

(b)

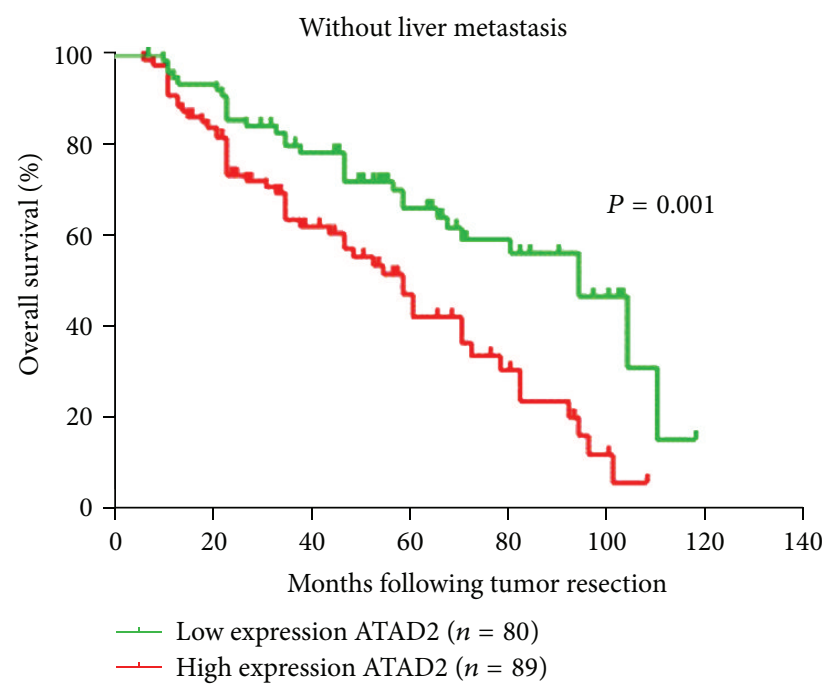

(d)

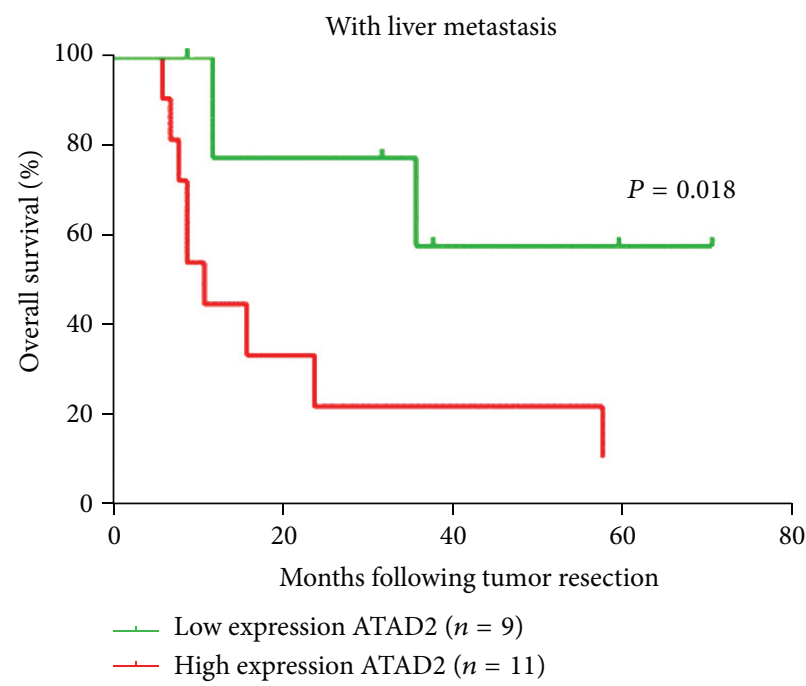

(e)

FIGURE 3: ATAD2 is correlated with overall survival rate in CRC patients. (a) Kaplan-Meier survival curves show high expression level of ATAD2 is significantly correlated with poor survival of CRC. (b)-(e) Correlation between ATAD2 expression and patient survival in colorectal cancer is independent of lymphatic metastasis and liver metastasis. $P$ values were calculated using the log-rank test. 
TABLE 1: Relationship between ATAD2 expression and clinicopathological features in 300 colorectal cancer patients.

\begin{tabular}{|c|c|c|c|}
\hline \multirow[b]{2}{*}{ Variable } & \multicolumn{3}{|c|}{$\operatorname{ATAD}(n)$} \\
\hline & $\begin{array}{c}\text { Low } \\
n=124\end{array}$ & $\begin{array}{c}\text { High } \\
n=176\end{array}$ & $P$ \\
\hline \multicolumn{4}{|l|}{ Age } \\
\hline$\leq 65$ years & $73(24.33)$ & $97(32.33)$ & \multirow{2}{*}{0.518} \\
\hline$>65$ years & $51(17.00)$ & $79(26.34)$ & \\
\hline \multicolumn{4}{|l|}{ Gender } \\
\hline Male & $68(22.37)$ & $99(32.57)$ & \multirow{2}{*}{0.978} \\
\hline Female & $56(18.42)$ & $81(26.64)$ & \\
\hline \multicolumn{4}{|l|}{ Tumor size } \\
\hline$\leq 5 \mathrm{~cm}$ & $78(26.00)$ & $72(24.00)$ & \multirow{2}{*}{$<0.001$} \\
\hline$>5 \mathrm{~cm}$ & $46(15.33)$ & $104(34.67)$ & \\
\hline \multicolumn{4}{|c|}{ Tumor location } \\
\hline Rectum & $73(24.33)$ & $103(34.33)$ & \multirow{2}{*}{0.873} \\
\hline Colon & $51(17.01)$ & $73(24.33)$ & \\
\hline \multicolumn{4}{|l|}{ Serum CEA } \\
\hline$\leq 5 \mathrm{ng} / \mathrm{mL}$ & $74(24.67)$ & $79(26.33)$ & \multirow{2}{*}{0.012} \\
\hline$>5 \mathrm{ng} / \mathrm{mL}$ & $50(16.67)$ & $97(32.33)$ & \\
\hline \multicolumn{4}{|c|}{ Lymph node metastasis } \\
\hline No & $53(17.67)$ & $52(17.33)$ & \multirow{2}{*}{0.018} \\
\hline N1-N2 & $71(23.67)$ & $124(41.33)$ & \\
\hline \multicolumn{4}{|c|}{ Liver metastasis } \\
\hline M0 & $109(36.33)$ & $137(45.66)$ & \multirow{2}{*}{0.025} \\
\hline M1 & $15(5.00)$ & $39(13.00)$ & \\
\hline \multicolumn{4}{|l|}{ Clinical stage } \\
\hline I & $17(5.67)$ & $12(4.00)$ & \multirow{4}{*}{0.004} \\
\hline II & $23(7.67)$ & $16(5.33)$ & \\
\hline III & $69(23.00)$ & $109(36.33)$ & \\
\hline IV & $15(5.00)$ & $39(13.00)$ & \\
\hline
\end{tabular}

group was distinctly better than that of the ATAD2 positive one for samples separated according to lymphatic metastasis and liver metastasis (Figures 3(b) $-3(\mathrm{e})$ ).

Furthermore, univariate and multivariate analyses were performed to confirm the possibility of ATAD2 used as an independent risk factor for poor prognosis in the 182 cases of CRC. Univariate Cox regression analyses showed that ATAD2 expression, tumor size, serum CEA, liver metastasis, and clinical stage were significantly associated with overall survival (OS) (Table 2). The multivariate Cox regression analysis confirmed ATAD2 expression, tumor size, and clinical stage as independent predictors of the OS in CRC patients (Table 2).

3.5. Effect of ATAD2 on CRC Cell Proliferation In Vitro. To better understand the biological function of ATAD2, we transfected siRNAs-ATAD2 into SW480 and HCT116 cells, and the expression levels of endogenous ATAD2 proteins were significantly suppressed by Western blot (Figure 4(a)). Moreover, we found that knockdown of ATAD2 resulted in a significant decrease in cell viability measured by CCK- 8 , compared with control (Figures 4(b)-4(c)).
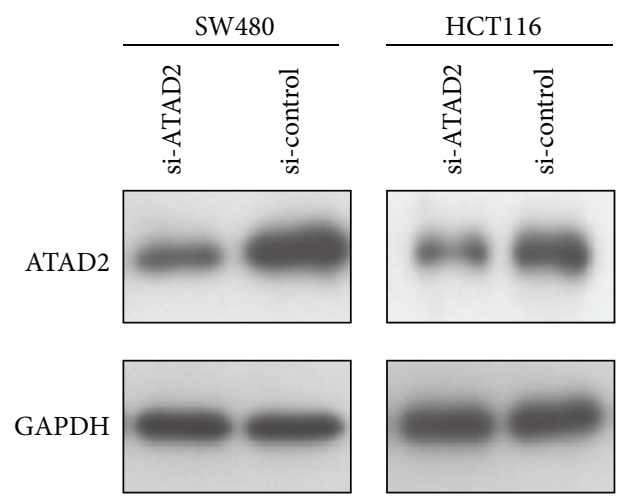

(a)

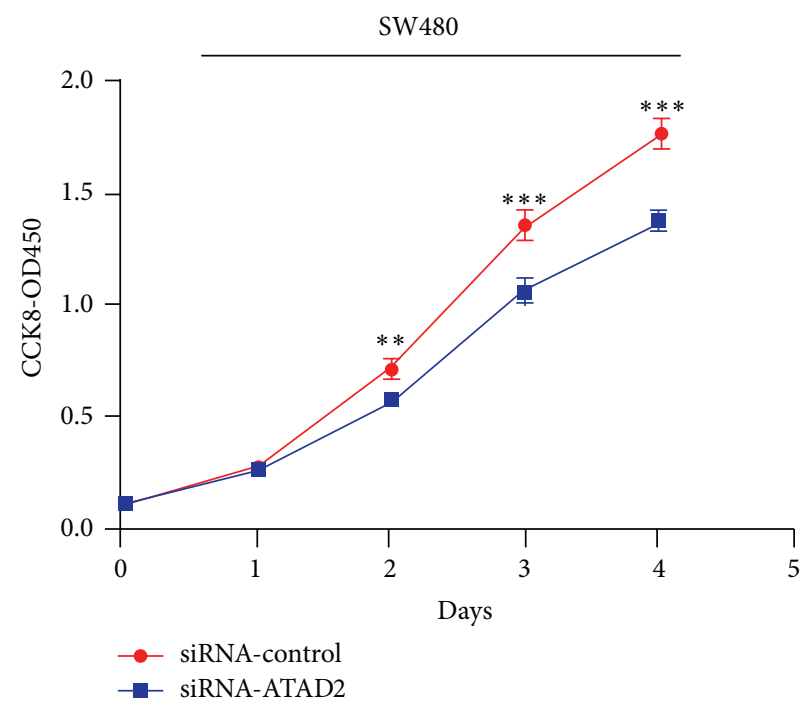

(b)

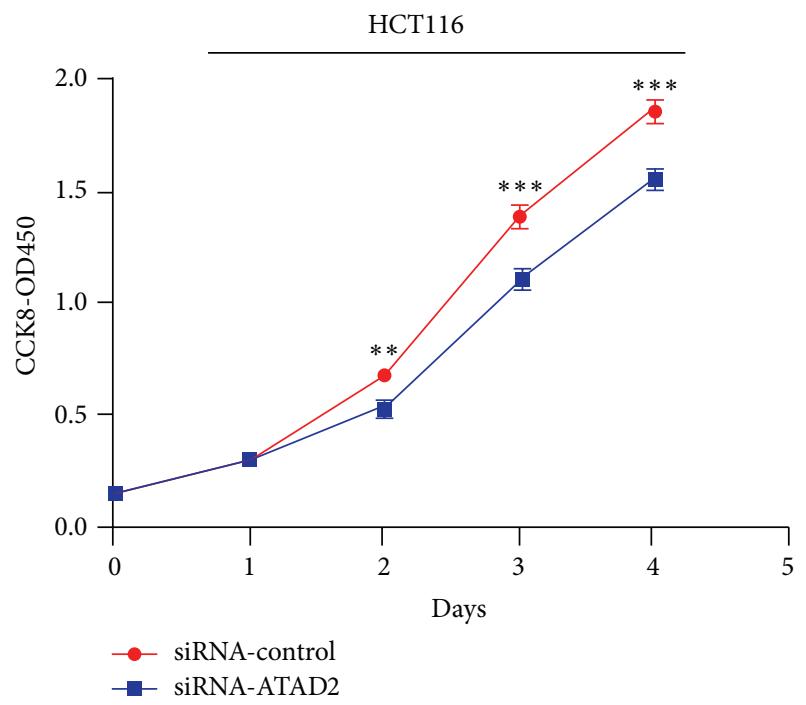

(c)

FIGURE 4: Effect of siRNA-ATAD2 on the proliferation of SW480 and HCT116 cells. (a) ATAD2 knockdown efficiency was confirmed by qPCR in SW480 and HCT116 cells. (b)-(c) siRNA-ATAD2 decreased cell viability measured by CCK8 assays. $P$ values were calculated by Student's $t$-test $\left({ }^{* *} P<0.01,{ }^{* * *} P<0.001\right)$ and the experiment was repeated at least three times. 
TABLE 2: Univariate and multivariate analyses showing the overall survival in colorectal cancer.

\begin{tabular}{|c|c|c|c|c|c|c|}
\hline \multirow{2}{*}{ Prognostic parameter } & \multicolumn{3}{|c|}{ Univariate analysis } & \multicolumn{3}{|c|}{ Multivariate analysis } \\
\hline & HR & $95 \% \mathrm{CI}$ & $P$ value & HR & $95 \% \mathrm{CI}$ & $P$ value \\
\hline ATAD2 (high versus low) & 2.272 & $1.479-3.491$ & 0.000 & 1.762 & $1.113-2.790$ & 0.016 \\
\hline Age $(>65$ versus $\leq 65)$ & 1.393 & $0.911-2.132$ & 0.127 & - & - & - \\
\hline Gender (male versus female) & 1.362 & $0.905-2.048$ & 0.139 & - & - & - \\
\hline Tumor size $(>5 \mathrm{~cm}$ versus $\leq 5 \mathrm{~cm})$ & 1.842 & $1.201-2.825$ & 0.005 & 1.698 & $1.075-2.681$ & 0.023 \\
\hline Tumor location (colon versus rectum) & 0.770 & $0.497-1.194$ & 0.243 & - & - & - \\
\hline Serum CEA $(>5 \mathrm{ng} / \mathrm{mL}$ versus $\leq 5 \mathrm{ng} / \mathrm{mL})$ & 1.628 & $1.063-2.493$ & 0.025 & 1.497 & $0.979-2.291$ & 0.063 \\
\hline Lymph node metastasis (present versus absent) & 1.686 & $1.099-2.588$ & 0.017 & 1.038 & $0.644-1.673$ & 0.879 \\
\hline Liver metastasis (present versus absent) & 3.308 & $1.777-6.151$ & 0.000 & 1.710 & $0.777-3.764$ & 0.183 \\
\hline Clinical stage (I vs. II vs. III vs. IV) & 1.983 & $1.549-2.539$ & 0.000 & 1.611 & $1.168-2.221$ & 0.004 \\
\hline
\end{tabular}

HR: hazard ratio; CI: confidence interval. The bold number represents the $P$ values with significant differences.

\section{Discussion}

Studies focused on human clinical specimens and genetically engineered mouse models of CRC have led to a better understanding of this genetic malignancy [22]. ATAD2 through different mechanisms broadly participated in various tumor types, such as prostate cancer, lung cancer, breast cancer, and ovarian cancer $[4,13,14,23]$. Previous studies indicated that ATAD2 directly interacted with the oncogene AIB1/ACTR and played an important role in the recruitment of ER $\alpha$ to promote the expression of genes driving cancer cell proliferation [24]. Furthermore, the ATPase domain of ATAD2 also enhanced the E2 induction of cyclin D1 and E2F1 expression [4]. In addition, ATAD2 was also directly associated with AR to activate AR-mediated transcription and was required to regulate the expression of androgen-induced genes that controlled cancer cell proliferation and survival [4].

In the current study, we firstly assessed ATAD2 expression at mRNA level. Both data from Oncomine database and our results showed that most primary CRC tissues exhibited significantly higher mRNA expression of ATAD2 than their matched normal tissues. This tendency was then confirmed by immunohistochemistry where 176 of 300 (58.67\%) CRC tissues were found to have high protein expression of ATAD2. Previous studies had demonstrated that overexpression of ATAD2 at the transcriptional level via miR-372 regulation promotes tumor malignancy and consequently results in poor outcome in liver adenocarcinoma patients [11]. Our results revealed similar phenomenon that ATAD2 has positive correlation with serum CEA level, lymph node metastasis, distant metastasis, and clinical stage in CRC. Besides, patients with a high level of ATAD2 expression had significantly shorter survival times compared to those with a low level of ATAD2 expression. Furthermore, univariate analysis showed that elevated ATAD2 expression was significantly associated with the OS of CRC patients; multivariate analysis demonstrated that ATAD2 expression, tumor size, and clinical stage are independent risk factors for the prognosis of CRC patients. Collectively, these results suggest that ATAD2 may be involved in the initiation and progression of CRC. Finally, we found that downexpression of ATAD2 could dramatically suppress the proliferation of SW480 and
HCT116 cells, which is in keeping with the previous studies on the other cancer cells. However, its underlying molecular mechanisms need to be further investigated.

In conclusion, our study demonstrated that ATAD2 expression was increased in CRC tissues compared to adjacent normal tissues and might be associated with pathological development of CRC. In addition, we found that high expression of ATAD2 could serve as an independent prognostic factor for CRC patients. Therefore, ATAD2 may be an important clinical marker of therapy for CRC.

\section{Conflict of Interests}

The authors declared that they have no competing interests.

\section{Authors' Contribution}

Yang Luo, Guang-Yao Ye, and Shao-Lan Qin contributed equally to this work.

\section{References}

[1] L.-A. Torre, F. Bray, R.-L. Siegel et al., "Global cancer statistics, 2012," Journal of Cancer Research and Clinical Oncology, vol. 65, no. 5, pp. 87-108, 2015.

[2] Y.-C. Lee, T.-C. Yin, Y.-T. Chen et al., "High expression of phospho-H2AX predicts a poor prognosis in colorectal cancer," Anticancer Research, vol. 35, no. 4, pp. 2447-2453, 2015.

[3] M. Ciró, E. Prosperini, M. Quarto et al., "ATAD2 is a novel cofactor for MYC, overexpressed and amplified in aggressive tumors," Cancer Research, vol. 69, no. 21, pp. 8491-8498, 2009.

[4] J. X. Zou, L. Guo, A. S. Revenko et al., "Androgen-induced coactivator ANCCA mediates specific androgen receptor signaling in prostate cancer," Cancer Research, vol. 69, no. 8, pp. 33393346, 2009.

[5] R. Beroukhim, C. H. Mermel, D. Porter et al., "The landscape of somatic copy-number alteration across human cancers," Nature, vol. 463, no. 7283, pp. 899-905, 2010.

[6] A. A. Alizadeh, M. B. Elsen, R. E. Davis et al., "Distinct types of diffuse large B-cell lymphoma identified by gene expression profiling," Nature, vol. 403, no. 6769, pp. 503-511, 2000. 
[7] X.-J. Ma, R. Salunga, J. T. Tuggle et al., "Gene expression profiles of human breast cancer progression," Proceedings of the National Academy of Sciences of the United States of America, vol. 100, no. 10, pp. 5974-5979, 2003.

[8] C. Caron, C. Lestrat, S. Marsal et al., "Functional characterization of ATAD2 as a new cancer/testis factor and a predictor of poor prognosis in breast and lung cancers," Oncogene, vol. 29, no. 37, pp. 5171-5181, 2010.

[9] S. V. Fernandez, F. M. Robertson, J. Pei et al., "Inflammatory breast cancer (IBC): clues for targeted therapies," Breast Cancer Research and Treatment, vol. 140, no. 1, pp. 23-33, 2013.

[10] J.-X. Zou, Z. Duan, J. Wang et al., "Kinesin family deregulation coordinated by bromodomain protein ANCCA and histone methyltransferase MLL for breast cancer cell growth, survival, and tamoxifen resistance," Molecular Cancer Research, vol. 12, no. 4, pp. 539-549, 2014.

[11] G. Wu, H. Liu, H. He et al., "miR-372 down-regulates the oncogene ATAD2 to influence hepatocellular carcinoma proliferation and metastasis," BMC Cancer, vol. 14, article 107, 2014.

[12] G. Wu, X. Lu, Y. Wang et al., "Epigenetic high regulation of ATAD2 regulates the Hh pathway in human hepatocellular carcinoma," International Journal of Oncology, vol. 45, no. 1, pp. 351-361, 2014.

[13] E.-V. Kalashnikova, A.-S. Revenko, A.-T. Gemo et al., "ANCCA/ATAD2 overexpression identifies breast cancer patients with poor prognosis, acting to drive proliferation and survival of triple-negative cells through control of B-Myb and EZH2," Cancer Research, vol. 70, no. 22, pp. 9402-9412, 2010.

[14] Y. Zhang, Y. Sun, Y. Li et al., "ANCCA protein expression is a novel independent poor prognostic marker in surgically resected lung adenocarcinoma," Annals of Surgical Oncology, vol. 20, supplement 3, pp. S577-S582, 2013.

[15] H. Murakami, S. Ito, H. Tanaka, E. Kondo, Y. Kodera, and H. Nakanishi, "Establishment of new intraperitoneal paclitaxelresistant gastric cancer cell lines and comprehensive gene expression analysis," Anticancer Research, vol. 33, no. 10, pp. 4299-4307, 2013.

[16] D. R. Rhodes, S. Kalyana-Sundaram, V. Mahavisno et al., "Oncomine 3.0: genes, pathways, and networks in a collection of 18,000 cancer gene expression profiles," Neoplasia, vol. 9, no. 2, pp. 166-180, 2007.

[17] K. Sugano, K. Maeda, H. Ohtani et al., "Expression of xCT as a predictor of disease recurrence in patients with colorectal cancer," Anticancer Research, vol. 35, no. 2, pp. 677-682, 2015.

[18] S. Kaiser, Y.-K. Park, J. L. Franklin et al., "Transcriptional recapitulation and subversion of embryonic colon development by mouse colon tumor models and human colon cancer," Genome Biology, vol. 8, no. 7, article R131, 2007.

[19] Y. Hong, T. Downey, K. W. Eu, P. K. Koh, and P. Y. Cheah, "A 'metastasis-prone' signature for early-stage mismatch-repair proficient sporadic colorectal cancer patients and its implications for possible therapeutics," Clinical and Experimental Metastasis, vol. 27, no. 2, pp. 83-90, 2010.

[20] M. Skrzypczak, K. Goryca, T. Rubel et al., "Modeling oncogenic signaling in colon tumors by multidirectional analyses of microarray data directed for maximization of analytical reliability," PLoS ONE, vol. 5, no. 10, Article ID e13091, 2010.

[21] F. Fan, S. Bellister, J. Lu et al., "The requirement for freshly isolated human colorectal cancer (CRC) cells in isolating CRC stem cells," British Journal of Cancer, vol. 112, no. 3, pp. 539-546, 2015.
[22] W.-N. Wan, Y.-X. Zhang, X.-M. Wang et al., "ATAD2 is highly expressed in ovarian carcinomas and indicates poor prognosis," Asian Pacific Journal of Cancer Prevention, vol. 15, no. 6, pp. 2777-2783, 2014.

[23] E.-Y. C. Hsia, E.-V. Kalashnikova, A.-S. Revenko, J. X. Zou, A. D. Borowsky, and H.-W. Chen, "Deregulated E2F and the $\mathrm{AAA}+$ coregulator ANCCA drive proto-oncogene ACTR/AIB1 overexpression in breast cancer," Molecular Cancer Research, vol. 8, no. 2, pp. 183-193, 2010.

[24] A.-S. Revenko, E.-V. Kalashnikova, A.-T. Gemo, J. X. Zou, and H.-W. Chen, "Chromatin loading of E2F-MLL complex by cancer-associated coregulator ANCCA via reading a specific histone mark," Molecular and Cellular Biology, vol. 30, no. 22, pp. 5260-5272, 2010. 


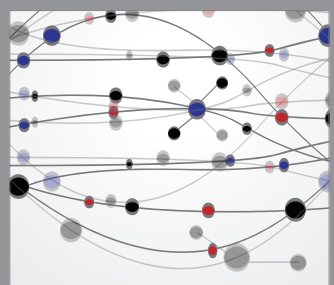

The Scientific World Journal
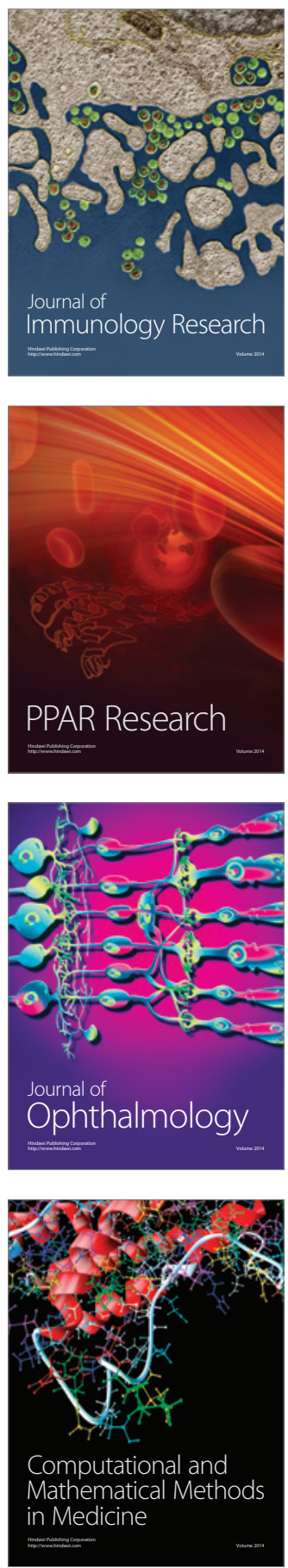

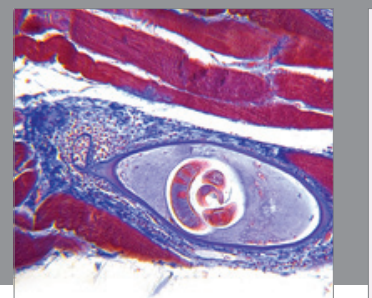

Gastroenterology

Research and Practice
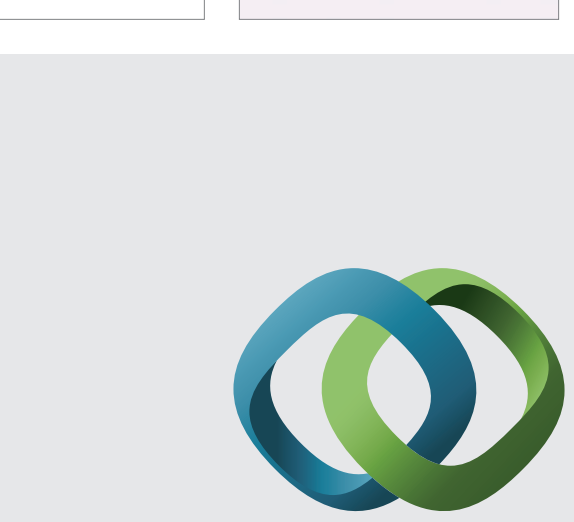

\section{Hindawi}

Submit your manuscripts at

http://www.hindawi.com
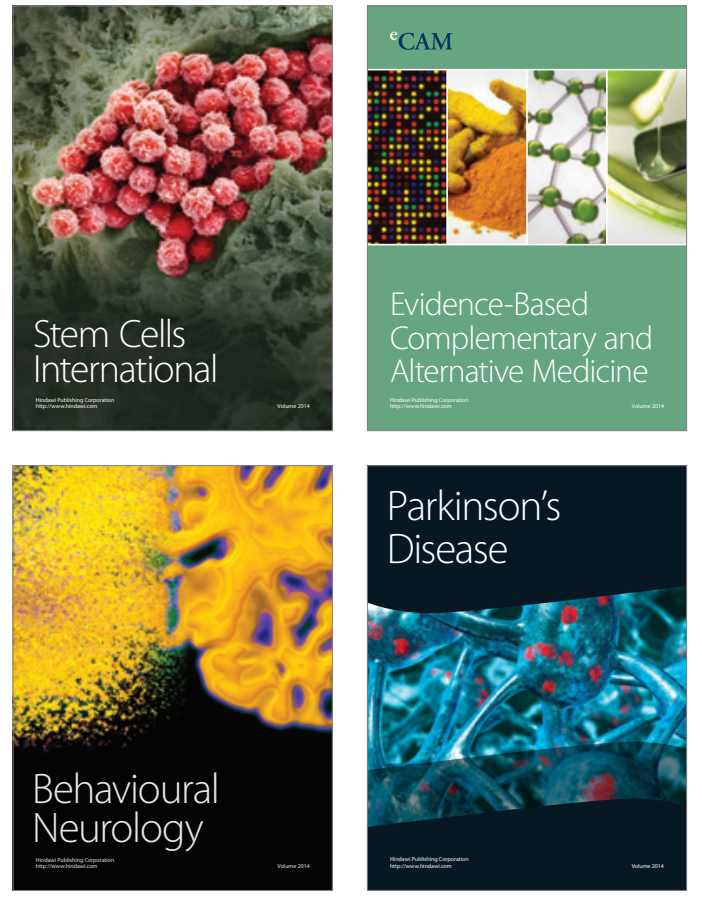
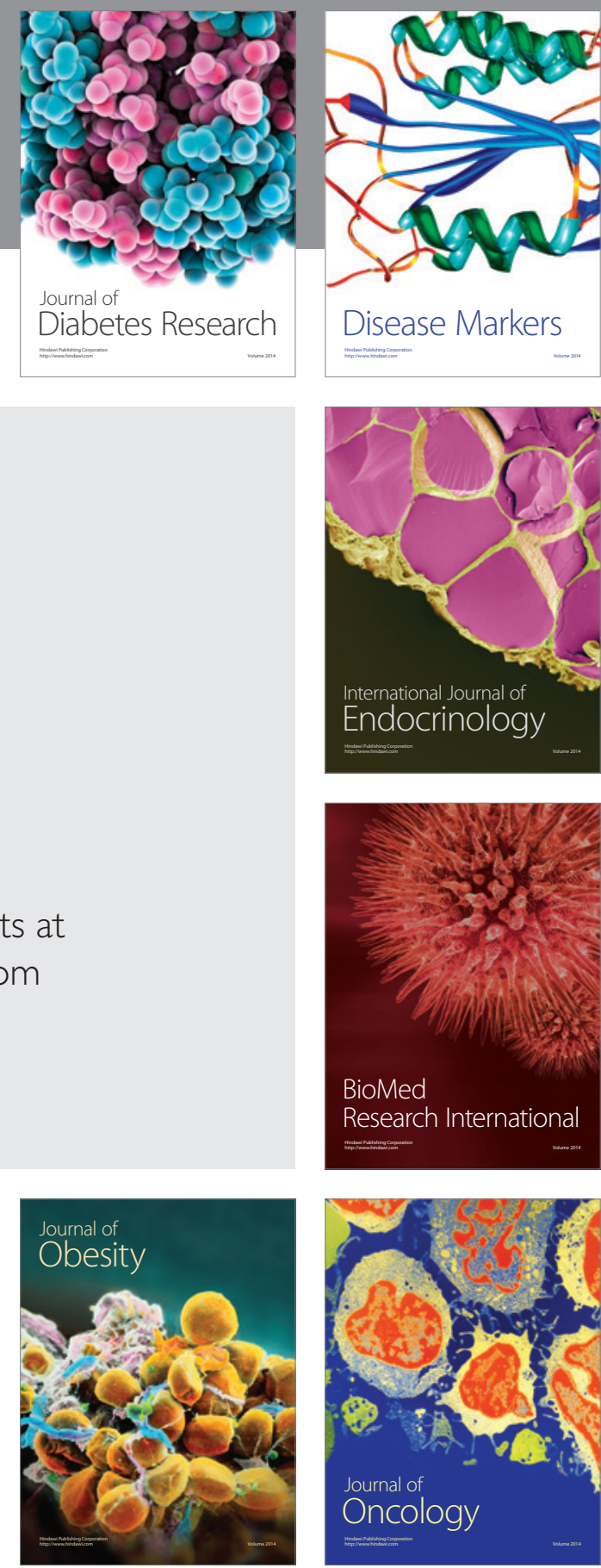

Disease Markers
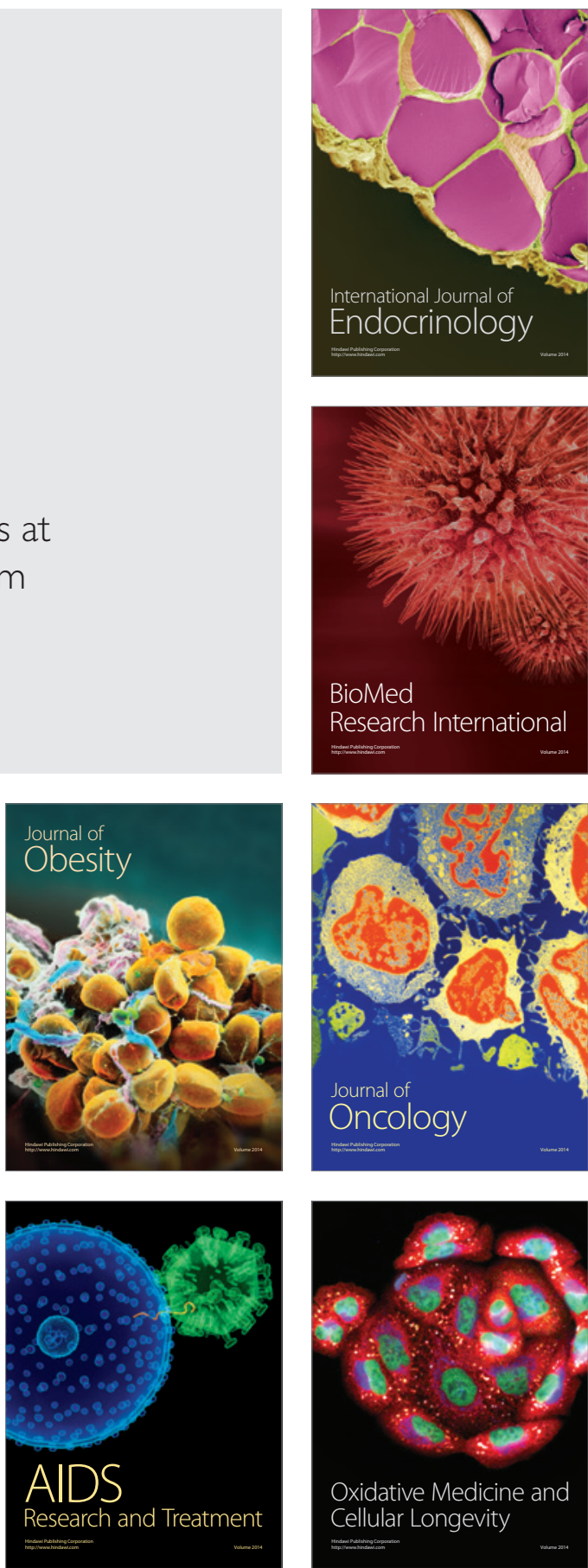